NOTICE: this is the author's version of a work that was accepted for publication in Solid State Ionics. Changes resulting from the publishing process, such as peer review, editing, corrections, structural formatting, and other quality control mechanisms may not be reflected in this document. Changes may have been made to this work since it was submitted for publication. A definitive version was subsequently published in Solid State lonics 264 (2014) 49; doi: 10.1016/j.ssi.2014.06.019 


\section{Trapping of oxide ions in $\delta-\mathrm{Bi}_{3} \mathrm{YO}_{6}$}

M. Krynski, ${ }^{1 *}$ W. Wrobel, ${ }^{1 *}$ C.E. Mohn, ${ }^{2}$ J. R. Dygas, ${ }^{1}$ M.Malys, ${ }^{1}$ F. Krok $^{1}$ and I. Abrahams ${ }^{3}$

${ }^{1}$ Faculty of Physics, Warsaw University of Technology, Koszykowa 75, 00-662 Warsaw, Poland.

${ }^{2}$ Department of Chemistry and Centre for Earth Evolution and Dynamics, University of Oslo, Postbox 1048 Blindern, N0316, Oslo, Norway

${ }^{3}$ Materials Research Institute, School of Biological and Chemical Sciences, Queen Mary University of London, Mile End Road, London E1 4NS, U.K.

*Corresponding author: W. Wrobel e-mail: wrobel@if.pw.edu.pl

M. Krynski e-mail: krynski.marcin@ outlook.com 


\begin{abstract}
A series of ab initio molecular dynamics (MD) simulations have been performed on the oxide ion conducting solid electrolyte $\delta-\mathrm{Bi}_{3} \mathrm{YO}_{6}$. The effect of different cationic neighbours on the residence time and occupancy of oxide ion sites was investigated. It was found that sites where neighbouring cations were exclusively bismuth exhibited the lowest residence time and lowest average site occupancy, with these parameters increasing with increasing number of yttrium cation neighbours. Residence time exhibited Arrhenius type temperature dependence, while the average site occupancies were almost temperature independent. The results are discussed in terms of oxide ion trapping by the dopant cation.
\end{abstract}

\title{
Keywords:
}

Bismuth oxide; bismuth yttrium oxide; fluorite structure, DFT calculations; molecular dynamics simulations. 


\section{Introduction}

The high temperature polymorph of bismuth oxide, $\delta-\mathrm{Bi}_{2} \mathrm{O}_{3}$, exhibits the highest known oxide ion conductivity in the order of $1 \mathrm{~S} \mathrm{~cm}^{-1}$ at $c a .1000 \mathrm{~K}$ [1]. However, below this temperature lower symmetry polymorphs of bismuth oxide are observed, $\alpha, \gamma$ and $\beta$ depending on the cooling rate [2]. All of these phases exhibit significantly lower ionic conductivity than the high temperature $\delta$-phase. The exceptionally high oxide ion conductivity of $\delta-\mathrm{Bi}_{2} \mathrm{O}_{3}$ is related to the high vacancy concentration in this phase and the high polarizability of the bismuth atoms.

The cubic $\delta$-phase can be preserved to room temperature through partial substitution of bismuth by a range of cations, most notably by those of the rare earth metals [3-7]. In the case of yttrium substitution, a wide solid solution range is obtained in the $\mathrm{Bi}_{2} \mathrm{O}_{3}-\mathrm{Y}_{2} \mathrm{O}_{3}$ system [5,8], with $\delta$-phase structures observed up to around $25 \%$ of substitution. These so called stabilized $\delta$-phases show very high oxide ion conductivities, over a range of temperatures, but at high temperatures all of them show lower conductivity than the parent $\delta-\mathrm{Bi}_{2} \mathrm{O}_{3}$, despite the fact that the isolvalent substitution in this system leads to compounds with nominally the same vacancy concentration. The type of substituting ion has a significant effect on ionic conductivity, not only by altering the size of the lattice and hence ion mobility, but also by altering the defect and electronic structure. For example, we have shown, using neutron diffraction analysis, that in $\delta-\mathrm{Bi}_{3} \mathrm{YO}_{6}$, Frenkel interstitial oxide ions are present, which are associated with the coordination polyhedra of the substituting yttrium cations, leading to higher vacancy concentrations on the fluorite tetrahedral sites [9].

$\delta-\mathrm{Bi}_{2} \mathrm{O}_{3}$ itself has been the subject of a number of computational studies including conventional molecular dynamics (MD), as well those based on density functional theory (DFT) [10-14]. However, relatively few of these studies are focused on transport properties. Aidhy et al. carried out conventional MD simulations to investigate the effect of polarizability on ion transport in $\delta-\mathrm{Bi}_{2} \mathrm{O}_{3}$ and have shown that high cation polarizability is a key factor in promoting high oxide ion diffusivity [15]. Mohn et al. [16-17] and Seko et al. [18] both calculated oxide ion diffusivity in $\delta$ $\mathrm{Bi}_{2} \mathrm{O}_{3}$ using DFT. Analysis of powder neutron diffraction data by maximum entropy methods (MEM) suggested that the diffusion pathways are in both $\langle 111\rangle$ and $\langle 100\rangle$ directions in $\delta-\mathrm{Bi}_{2} \mathrm{O}_{3}$ [19], as well as in the ytterbium substituted system $\delta-\mathrm{Bi}_{1.4} \mathrm{Yb}_{0.6} \mathrm{O}_{3}$ [20]. Zhong et al. [21] using ab initio calculations have studied the influence of dopant ions on the activation energy for ionic transport and reported that activation energy increases with increasing dopant concentration, 
although in the case of $\mathrm{Y}$ substitution they noted an initial decrease in activation energy at lower levels of substitution. Payne et al. [22] have shown in the rhenium substituted system, $\mathrm{Bi}_{28} \mathrm{Re}_{2} \mathrm{O}_{49}$ (a tetragonally ordered fluorite), that it is possible, using DFT methods, to distinguish oxide ion movements around different cations. In this case significant differences were seen in the oxide ion movements around rhenium and bismuth.

In the present study, DFT calculations are utilized to examine oxide ion diffusion in $\delta-\mathrm{Bi}_{3} \mathrm{YO}_{6}$. In particular, this study focuses on characterizing the influence of the substituent cation, $\mathrm{Y}^{3+}$, on oxide ion transport in this system.

\section{Methodology}

DFT calculations were carried out within a $2 \times 2 \times 2$ supercell (80 ions in total) of $\delta-\mathrm{Bi}_{3} \mathrm{YO}_{6}$ using the Vienna Ab-initio Simulation Package (VASP) and a plane wave basis set [23,24]. The exchange-correlation function was treated within the generalized gradient approximation of Perdew-Burke-Ernzerhof [25] and the electron-ion interactions were described by the full potential projector augmented wave method [26], with 5, 6 and 11 valence electrons for $\mathrm{Bi}, \mathrm{O}$ and $\mathrm{Y}$ respectively (including $4 s$ and $4 p$ electrons for the later). Forces were calculated according to the Hellmann-Feynman theorem [27,28] and the kinetic energy was controlled by means of the NoseHoover thermostat $[29,30]$. The plane wave cutoff energy was $450 \mathrm{eV}$ for all calculations. The sampling of the Brillouin zone was performed using the Monkhorst-Pack scheme [31] at the gamma point. All calculations were performed under periodic boundary conditions. The MD simulations were performed from initially relaxed structures. The global break point for the electronic loop was set to $10^{-5} \mathrm{eV} /$ cell. The crystallographic model and initial lattice parameters for $\delta$ - $\mathrm{Bi}_{3} \mathrm{YO}_{6}$ were obtained from the neutron scattering analysis [9] at $1073 \mathrm{~K}$. Bismuth and yttrium atoms were placed at the $4 a$ crystallographic sites of the $F m-3 m$ space group, whereas oxygen atoms and oxide ion vacancies were distributed quasi randomly over the $8 c$ sites, maintaining two oxide ion vacancies per unit cell.

To ensure no implied cation ordering, the unit cell was shifted by $(1 / 4,1 / 4,1 / 4)$ and the yttrium atoms were placed quasi randomly, with one yttrium ion per unit cell. Additionally three parallel runs with three different atom configurations were performed to ensure not only randomness of ion 
distribution, but also to improve the statistics of ion jumps during ab initio molecular dynamics (AIMD). Pair distribution functions (PDF) determined for all three parallel runs over the total time of the MD simulation compare well with the experimentally curve determined from neutron scattering experiments [9], justifying the statistically averaged atom distribution of the solid solution (Fig. 1). The MD simulations were carried out at 973, 1073, 1173 and 1273 K. Calculations were performed over a total of $70 \mathrm{ps}$ in steps of $10 \mathrm{fs}$, but to minimize the influence of initial thermodynamic relaxation of the system, the data corresponding to the first $10 \mathrm{ps}$ were not taken into account for further analysis. The time step used in the present study has previously been shown to give equivalent accuracy in the calculations compared to shorter time steps, provided a suitable cutoff energy is used [17].

To capture the oxide ion jump trajectories, high frequency oscillations of the oxide ions were excluded from calculations using a low pass Chebyshev filter. The cutoff frequency for the Chebyshev filter was determined based on the Fourier analysis of the ionic trajectories. For the frequencies above $5 \times 10^{12} \mathrm{~Hz}$, only thermal vibrations are observed. The residence time for oxide ions is defined as the time spent by the ion in a given tetrahedral cavity. Since there is a distance of 0.5 unit cells between the neighbouring crystallographic $8 c$ positions, any oxide ion movement over a distance longer than 0.25 unit cells was considered to be an ion jump, provided the time spent at the destination was longer than $0.1 \mathrm{ps}$. This value is considered to be sufficient time to allow more than one full vibration at the destination. Fig. 2a shows a typical variation of one of the coordinates for a single oxide ion over 60 ps simulation time. The ion is considered to have entered the site at point $i$. It then oscillates around $y=0.75$ for around $25 \mathrm{ps}$. At point $j$ the ion crosses the threshold to next site, but immediately returns. It is only after passing point $k$ that the ion is considered to have jumped to a neighbouring site, where it resides for some time. When calculating average residence times without a Chebyshev filter, a value is obtained that is unrealistically short, by taking the average of times $j-i$ and $k-j$. A more realistic estimation of the residence time is obtained by application of a Chebyshev filter, with a suitable cutoff frequency. This removes short time oscillations, giving a residence time equal to the difference between points $i$ and $k$. Since the applied filter removes all high frequencies related to thermal vibrations, the trajectories of moving ions become more visible (Fig. 2b). However, the applied filter does not affect the mean square displacement (Fig. 3) and the diffusion coefficient calculated for the raw and processed data is virtually the same. 


\section{Results and discussion}

The oxide ion pathways in $\delta-\mathrm{Bi}_{3} \mathrm{YO}_{6}$, obtained in the MD simulation, were examined to reveal details of the conduction mechanism. Table 1 summarizes the statistics for oxygen ion jumps at the studied temperatures. More than $94 \%$ of oxide ion jumps were seen to occur in the $<100>$ crystallographic direction. This strong preference for the $<100\rangle$ jump direction is also seen in the parent compound $\delta-\mathrm{Bi}_{2} \mathrm{O}_{3}[17]$.

Since the ion movements are related to their interactions with their surroundings, the observed $<100>$ jump direction preference could be explained in terms of energy barriers for ion movement in different crystallographic directions. For this purpose the energy distribution landscape was derived from the ionic density map assuming a Boltzmann distribution of ion positions:

$$
N_{x}=N_{0} \cdot e^{-\frac{\Delta E}{k T}}
$$

where $N_{x}$ represents the number of times that oxide ions were present at position $x, N_{0}$ is the number of times that oxide ions were present at the point of highest ionic density (usually close to the centre of the tetrahedral site), $k$ is the Boltzmann constant, $T$ is the temperature and the $\Delta E$ stands for the energy difference between position $x$ and the highest ionic density point. Fig. 4 shows a <110> section through the anion energy distribution landscape for $\delta-\mathrm{Bi}_{3} \mathrm{YO}_{6}$, determined for the MD calculations at $1073 \mathrm{~K}$ according to above procedure. The energy barrier for oxide ion jumps in the $<100>$ direction is significantly smaller than that for jumps in the $<110\rangle$ direction $(0.44 \mathrm{eV}$ and $0.86 \mathrm{eV}$ respectively) and reflects the observed preferred jump direction. It is noteworthy that the energy barrier in the $\langle 100\rangle$ direction determined with this approach is comparable with the activation energy previously determined for the same system from Mean Square Displacement (MSD) analysis [33]. A similar approach was used by Kushima and Yildiz [32] for the hetero interfacial system, YSZ/SrTiO 3 , to correlate energy barriers with the number of successful jumps.

In order to investigate the influence of yttrium ions on the oxide ion transport mechanism, the simulated supercell was divided into 64 equally sized compartments, equivalent to an octant of the Fm-3m subcell, centred on the $8 c$ oxygen site (Fig. 5). There are four cations located on the corners of each of octant and depending on the number of yttrium ions, $n$, in the octant, four types of octants were considered with $n=0,1,2$ and 3 . The case of $n=4$ was ignored as it was considered to be 
extremely rare. The number of octants $\left(N_{n}\right)$ of each type $n$, observed in the present study are listed in Table 2.

The oxide ion occupancy of each octant, $f_{w}^{n}(t)$, at time $t$ of the simulation was calculated as an average value over $N_{n}$ and time window $w$ according to the formula:

$$
f_{w}^{n}(t)=\frac{1}{N_{n}} \sum_{i=1}^{N_{n}} \frac{1}{w} \sum_{s=0}^{w} O c c_{i}^{n}(t-s)
$$

where $\operatorname{Occ}_{i}^{n}(t-s)$ is the occupancy of the oxide ion site in octant $i$ of type $n$ at time $t-s$ and has a value of 0 if the site is unoccupied and 1 or more if the site is occupied by one or more oxide ions, respectively.

The time dependence of the average occupancies calculated in the time window $w=1 \mathrm{ps}, f_{w=1}^{n}(t)$, for different types of octants is presented in Fig. 6. It is evident that $f_{w=1}^{n}$ increases with increasing value of $n$. In the case of octants containing no yttrium ions $(n=0)$, the value of $f_{w=1}^{n}$ is significantly lower than the stoichiometric value of 0.75 , while octants with $n=1,2$ and 3 show $f_{w=1}^{n}$ values higher than the stoichiometric value. It should be noted, that since there are only 3 octants of type $n=3$ in the model, the standard deviation of $f_{w=1}^{n}$ for this type of octant is large and for clarity is not presented in Fig. 6.

To determine the convergence of both average site occupancies and the standard deviation of the average site occupancies, a different type of analysis was applied. The average oxide ion site occupancy for the octants of type $n$ at time $t, f_{w=t}^{n}(t)$, was calculated as an average value over the time window from the beginning of the simulation to time $t$. The obtained values for different types of octants at $1073 \mathrm{~K}$ are presented in Fig. 7. Significant variations of the $f_{w=t}^{n}(t)$ value and standard deviation of this quantity, observed within first 15 ps of simulation, are explained by the poor statistics (small number of ion jumps) within this time window. Indeed, irrespective of the point of origin of analysis, sufficient data points must be analysed to allow convergence of the standard deviation. In order to allow for more rapid convergence, data from three parallel runs were analysed. After $c a$. 30 ps both $f_{w=t}^{n}(t)$ and its standard deviation converged. 
Where the time window is the full duration of the simulation $(w=60 \mathrm{ps})$, the standard deviation of $f_{w=60}^{n}$, is $c a .2-5 \%$ and is consistent with values reported for DFT calculations of $\delta-\mathrm{Bi}_{2} \mathrm{O}_{3}$ [18]. It is noteworthy that the standard deviation of the average occupancy over all types of octants is $c a$. $20 \%$ as reported previously [33], i.e. this value reflects the variety of oxygen surroundings, rather than instability of the system. Table 3 summarizes $f_{w=60}^{n}$ values at the four investigated temperatures. As seen for $f_{w=1}^{n}, f_{w=60}^{n}$ increases with increasing value of $n$ and this trend is almost temperature independent.

The total average residence time, $\tau$, as well as the residence time for oxide ions in the different types of octants $\tau_{n}$ was investigated. As discussed previously, to ensure that the short time jumps (jumprelaxation events) do not affect the calculated average residence times, a low pass Chebyshev filter was applied. The obtained results are summarized graphically in Fig. 8. The average residence time for oxide ions is seen to increase with increasing number of yttrium ions per octant $(n)$ at all studied temperatures. The residence time for oxide ions in octants containing only bismuth cations $(n=0)$ at $1073 \mathrm{~K}$ is comparable with the value of 8 ps reported for $\delta-\mathrm{Bi}_{2} \mathrm{O}_{3}$ at $1023 \mathrm{~K}$ [17]. In the case of octants with three yttrium ions $(n=3)$, the residence time for oxide ions is nearly four times longer than in the case of $n=0$.

With the exception of $n=3$, where the statistics are poor, the average residence time decreases with increasing temperature for all octant types. The temperature dependence of the inverse of the average total residence time $1 / \tau$ (which is an indication of oxide ion jump frequency and is proportional to the diffusion coefficient) over all octant types is presented in the form of an Arrhenius type plot, with plots for individual octant types given in Fig. 9. The highest average value for $1 / \tau$ is observed for the $n=0$ octants and is an indication of higher mobility of oxide ions surrounded only by bismuth cations, compared to those with yttrium neighbours. Thus yttrium ions can be considered to act as trapping centres for mobile oxide ions. Similar conclusions can be made when analysing the number of jumps to and from octants of type $n, C_{n}$. The obtained results at 1173 $\mathrm{K}$ are presented in Table 2 and show that the majority of oxide ion jumps observed during MD simulations occur in bismuth rich octants, with around 5 times fewer jumps involving $n=3$ octants.

We have previously reported the diffusion coefficient for $\mathrm{Bi}_{3} \mathrm{YO}_{6}[33]\left(\mathrm{D}=0.25 \times 10^{-5} \mathrm{~cm}^{2} \mathrm{~s}^{-1}\right.$ at $1073 \mathrm{~K})$ to be lower than the one determined for $\delta-\mathrm{Bi}_{2} \mathrm{O}_{3}$ [17] $\left(\mathrm{D}=1.0 \times 10^{-5} \mathrm{~cm}^{2} \mathrm{~s}^{-1}\right.$ at $\left.1033 \mathrm{~K}\right)$. Considering the higher occupancy and longer residence times in the yttrium rich surroundings, the 
lower diffusion and conductivity in $\mathrm{Bi}_{3} \mathrm{YO}_{6}$ can be associated with the presence of yttrium ions in the system. This effect is attributed mainly to differences in the electronic structure of bismuth and yttrium, especially the lack of the stereochemically active lone pair in the latter case. The electronic orbital associated with the bismuth $6 \mathrm{~s}^{2}$ electrons is thought to have a major influence on the high oxide ion conductivity of bismuth oxide [15] and in the case of $\mathrm{Bi}_{3} \mathrm{YO}_{6}$ it has been shown to be directed towards oxide ion vacancies [33], promoting their movement.

\section{Conclusions}

MD calculations for $\delta-\mathrm{Bi}_{3} \mathrm{YO}_{6}$ show that the majority of oxide ion jumps occur in the <100> crystallographic direction. The average occupancy of and residence times in oxide ion sites with neighbouring yttrium ions is significantly higher than for those sites with exclusively bismuth neighbours. This is expected to result in low values of oxide ion diffusion for oxide ions in yttrium rich areas. The majority of ion jumps are observed to and from oxide ion sites with two or more bismuth neighbours. The results are consistent with the concept of oxide ion trapping in the vicinity of the yttrium dopant ions in $\delta-\mathrm{Bi}_{3} \mathrm{YO}_{6}$. This phenomenon appears to be almost temperature independent over the range 973 to $1273 \mathrm{~K}$. Trapping of oxide ions in the vicinity of yttrium ions in $\delta-\mathrm{Bi}_{3} \mathrm{YO}_{6}$ explains the lower conductivity of this compound compared to that in $\delta-\mathrm{Bi}_{2} \mathrm{O}_{3}$ at high temperatures. This trapping effect is related to differences in the electronic structure of bismuth and yttrium, especially the lack of the stereochemically active $6 s^{2}$ lone pair in the latter case.

Acknowledgements

We gratefully acknowledge the National Science Centre Poland for project grant numbers 2012/05/E/ST3/02767 and 2013/09/N/ST3/04326

\section{References}

1. T. Takahashi, H. Iwahara, Y. Nagaj, J. Appl. Electrochem. 2 (1972) 97.

2. H.A. Harwig, Z. Anorg. Allg. Chem. 444 (1978) 151. 
3. G. Mairesse, B. Scrosati, A. Magistris, C.M. Mari, G. Mariotto (Eds.), Kluwer Academic Publishers, Dordrecht, 1993, p271.

4. P. Shuk, H.D. Wiemhöfer, U. Guth, W. Göpel, M. Greenblatt, Solid State Ionics 89 (1996) 179.

5. N.M. Sammes, G.A. Tompsett, H. Näfe, F. Aldinger, J. Eur. Ceram. Soc. 19 (1999) 1801.

6. A.M. Azad, S. Larose, S.A. Akbar, J. Mater. Sci. 29 (1994) 4135.

7. M. Drache, P. Roussel, J-P. Wignacourt, Chem. Rev. 107 (2007) 80.

8. R.K. Datta, J.P. Meehan, Z. Anorg. Allg. Chem. 383 (1971) 328.

9. I. Abrahams, X. Liu, S. Hull, S.T. Norberg, F. Krok, A. Kozanecka-Szmigiel, M.S. Islam, S.J. Stokes, Chem. Mater. 22 (2010) 4435.

10. P.W.M. Jacobs, D.A. MacDonaill, Solid State Ionics 18\&19 (1986) 209.

11. P.W.M. Jacobs, D.A. MacDonaill, Solid State Ionics 23 (1987) 279.

12. P.W.M. Jacobs, D.A. MacDonaill, Solid State Ionics 23 (1987) 295.

13. P.W.M. Jacobs, D.A. MacDonaill, Solid State Ionics 23 (1987) 307.

14. N.I. Medvedeva, V.P. Zhukov, V.A. Gubanov, D.L. Novikov, M.L. Klein, J. Phys. Chem. Solids 57 (1996) 1243.

15. D.S. Aidhy, J.C. Nino, S.B. Sinnott, E.D. Wachsman, S.R. Phillpot, J. Am. Ceram. Soc.

91 (2008) 2349.

16. C.E. Mohn, S. Stølen, S.T. Norberg, S. Hull, Phys. Rev. Lett. 102 (2009) 155502.

17. C.E. Mohn, S. Stølen, S.T. Norberg, S. Hull, Phys. Rev. B 80 (2009) 024205.

18. A. Seko, Y. Koyama, A. Matsumoto, I. Tanaka, J. Phys.: Condens. Matter 24 (2012) 475402.

19. M. Yashima, D. Ishimura, Chem. Phys. Lett. 378 (2003) 395.

20. M. Yashima, D. Ishimura, Appl. Phys. Lett. 87 (2005) 221909.

21. G. H. Zhong, J. L. Wang, Z. Zeng, J. Phys.: Conf. Ser. 29 (2006) 106.

22. J. L. Payne, J. D. Farrell, A. M. Linsell, M. R. Johnson, I. Radosavljević Evans, Solid State Ionics 244 (2013) 35-39.

23. G. Kresse, J. Furthmüller, Phy. Rev. B 54 (1996) 11169. 
24. G. Kresse, J. Furthmüller, Comp. Mater. Sci. 6 (1996) 15.

25. J. Perdew, K. Burke, M. Ernzerhof, Phys. Rev. Lett. 77 (1996) 3865.

26. G. Kresse, J. Joubert, Phys. Rev. B 59 (1999) 1758.

27. H. Hellmann, Einführung in "Die Quantenchemie, Franz Deuticke”, Leipzig, 1937.

28. R. Feynman, Phys. Rev. 56 (1939) 340.

29. S. Nosé, J. Chem. Phys. 81 (1984) 511.

30. W. Hoover, Phys. Rev. A 31, 1695 (1985).

31. H.J. Monkhorst, J.D. Pack, Phys. Rev. B 13 (1976) 5188.

32. A. Kushima, B. Yildiz, J. Mater. Chem. 20 (2010) 4809.

33. M. Krynski, W. Wrobel, J.R. Dygas, J. Wrobel, M. Malys, P. Śpiewak, K.J. Kurzydlowski, F. Krok, I. Abrahams, Solid State Ionics 245-246 (2013) 43. 
Table 1. Statistical analysis of oxide ion jump directions at different temperatures. Estimated errors were calculated as $(100 \%) /($ total number of jumps).

\begin{tabular}{|c|c|c|c|c|}
\hline Jump direction & $973 \mathrm{~K}$ & $1073 \mathrm{~K}$ & $1173 \mathrm{~K}$ & $1273 \mathrm{~K}$ \\
\hline$<100>$ & $95.90 \pm 0.11 \%$ & $95.1 \pm 0.8 \%$ & $93.4 \pm 0.6 \%$ & $94.2 \pm 0.5 \%$ \\
\hline$<110>$ & $4.10 \pm 0.11 \%$ & $4.3 \pm 0.8 \%$ & $6.2 \pm 0.6 \%$ & $5.2 \pm 0.5 \%$ \\
\hline$<111>$ & $<0.10 \pm 0.11 \%$ & $0.5 \pm 0.8 \%$ & $0.4 \pm 0.6 \%$ & $0.4 \pm 0.5 \%$ \\
\hline
\end{tabular}

Table 2. Summary of oxide ion jumps in a $2 \times 2 \times 2$ super cell of $\delta-\mathrm{Bi}_{3} \mathrm{YO}_{6}$ over $60 \mathrm{ps}$, where $C_{n}$ is the number of oxide ion jumps involving different octant types $n$ and $N_{n}$ is the number of octants of each type.

\begin{tabular}{|c|c|c|c|}
\hline$n$ & $N_{n}$ & $C_{n}$ & $C_{n} / N_{n}$ \\
\hline 0 & 18 & 173 & 9.6 \\
\hline 1 & 31 & 194 & 6.3 \\
\hline 2 & 12 & 76 & 6.3 \\
\hline 3 & 3 & 6 & 2.0 \\
\hline
\end{tabular}

Table 3. Average occupancy $f_{w=60}^{n}$ of octants of each type calculated as an average value over 60 ps of MD simulation.

\begin{tabular}{|c|c|c|c|c|}
\hline$n$ & $973 \mathrm{~K}$ & $1073 \mathrm{~K}$ & $1173 \mathrm{~K}$ & $1273 \mathrm{~K}$ \\
\hline 0 & $0.66 \pm 0.04$ & $0.61 \pm 0.02$ & $0.64 \pm 0.02$ & $0.65 \pm 0.03$ \\
\hline 1 & $0.74 \pm 0.03$ & $0.79 \pm 0.02$ & $0.78 \pm 0.01$ & $0.77 \pm 0.01$ \\
\hline 2 & $0.90 \pm 0.02$ & $0.82 \pm 0.01$ & $0.84 \pm 0.03$ & $0.81 \pm 0.02$ \\
\hline 3 & $0.86 \pm 0.34$ & $0.92 \pm 0.08$ & $0.86 \pm 0.08$ & $0.91 \pm 0.05$ \\
\hline
\end{tabular}




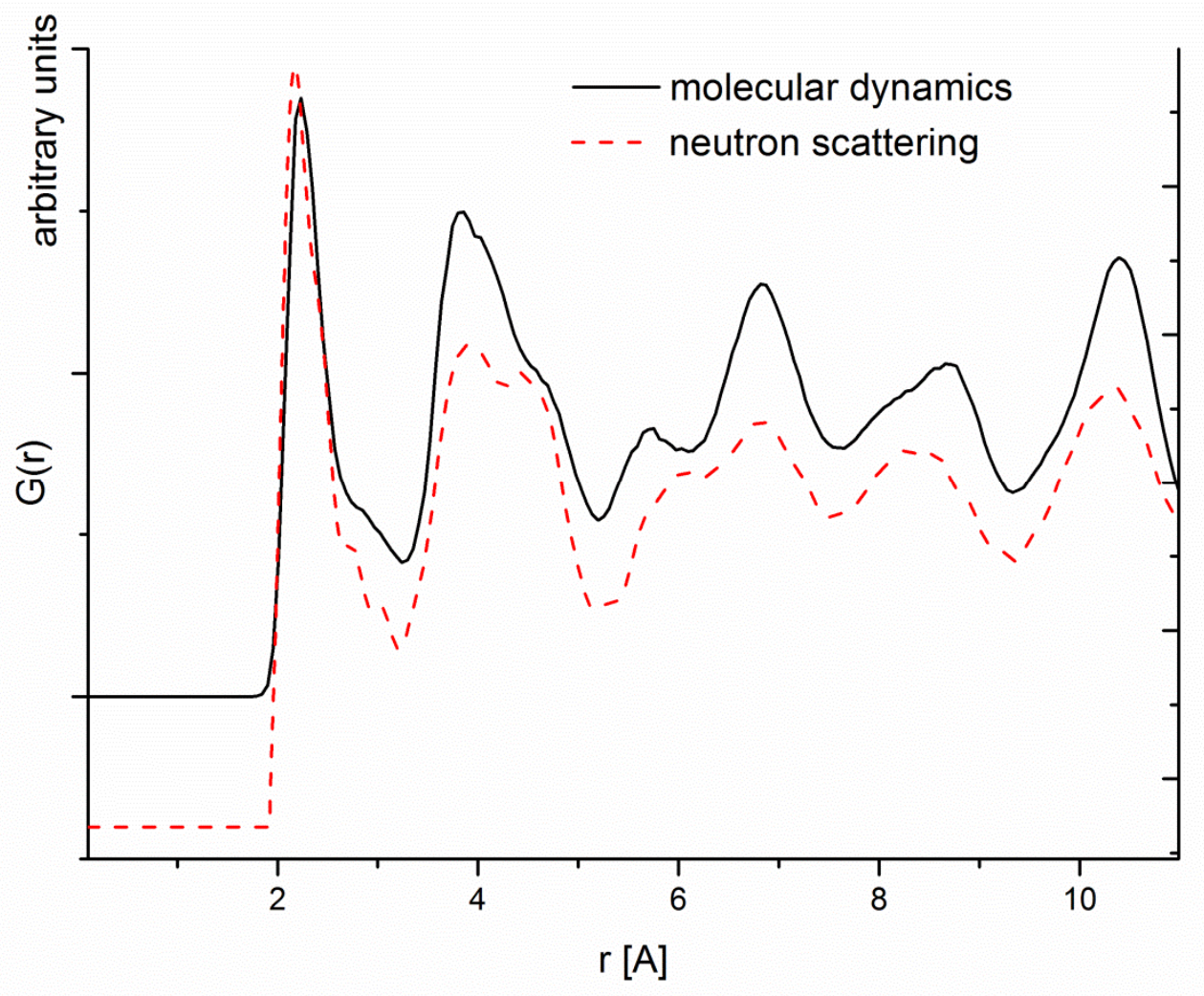

Fig.1. Pair distribution function (PDF) for $\delta-\mathrm{Bi}_{3} \mathrm{YO}_{6}$ determined from $\mathrm{MD}$ simulation of three parallel runs over the total simulation time at $1073 \mathrm{~K}$, compared to the experimentally derived data from neutron diffraction measurement [9]. 

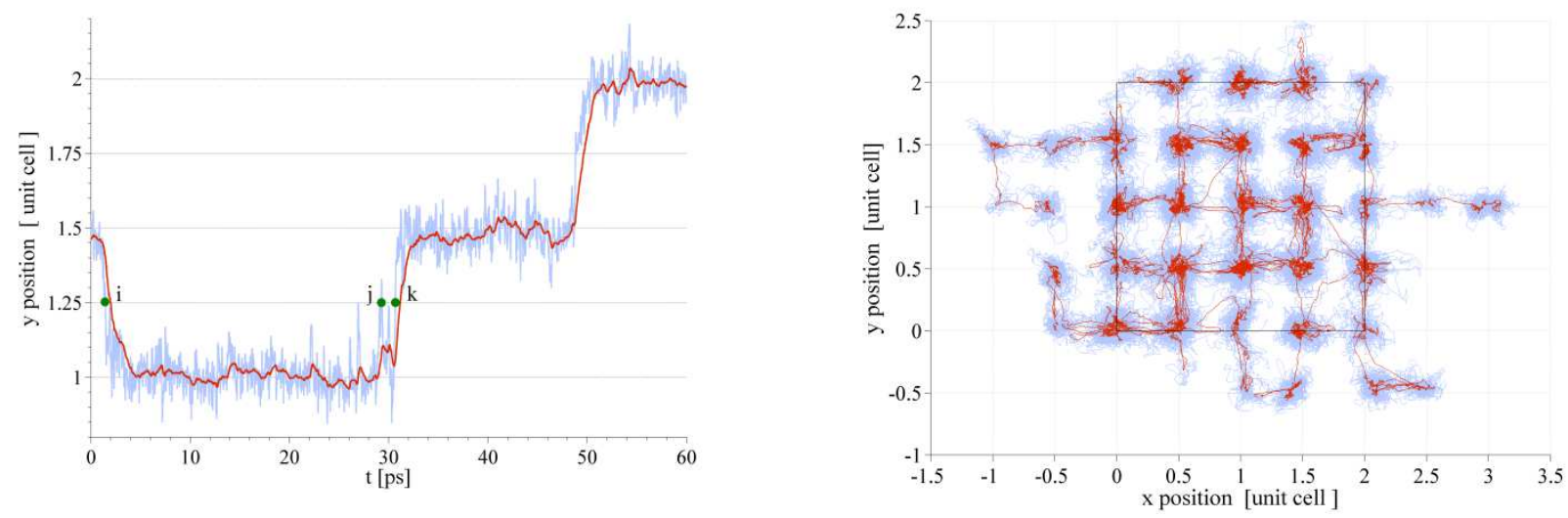

Fig. 2. (a) Typical time evolution of $y$ coordinate for a single oxide ion and (b) projection of oxide ion movements in the $\langle 110\rangle$ plane. The blue lines represent unfiltered data and the red lines represent data after processing with a low-pas filter. 


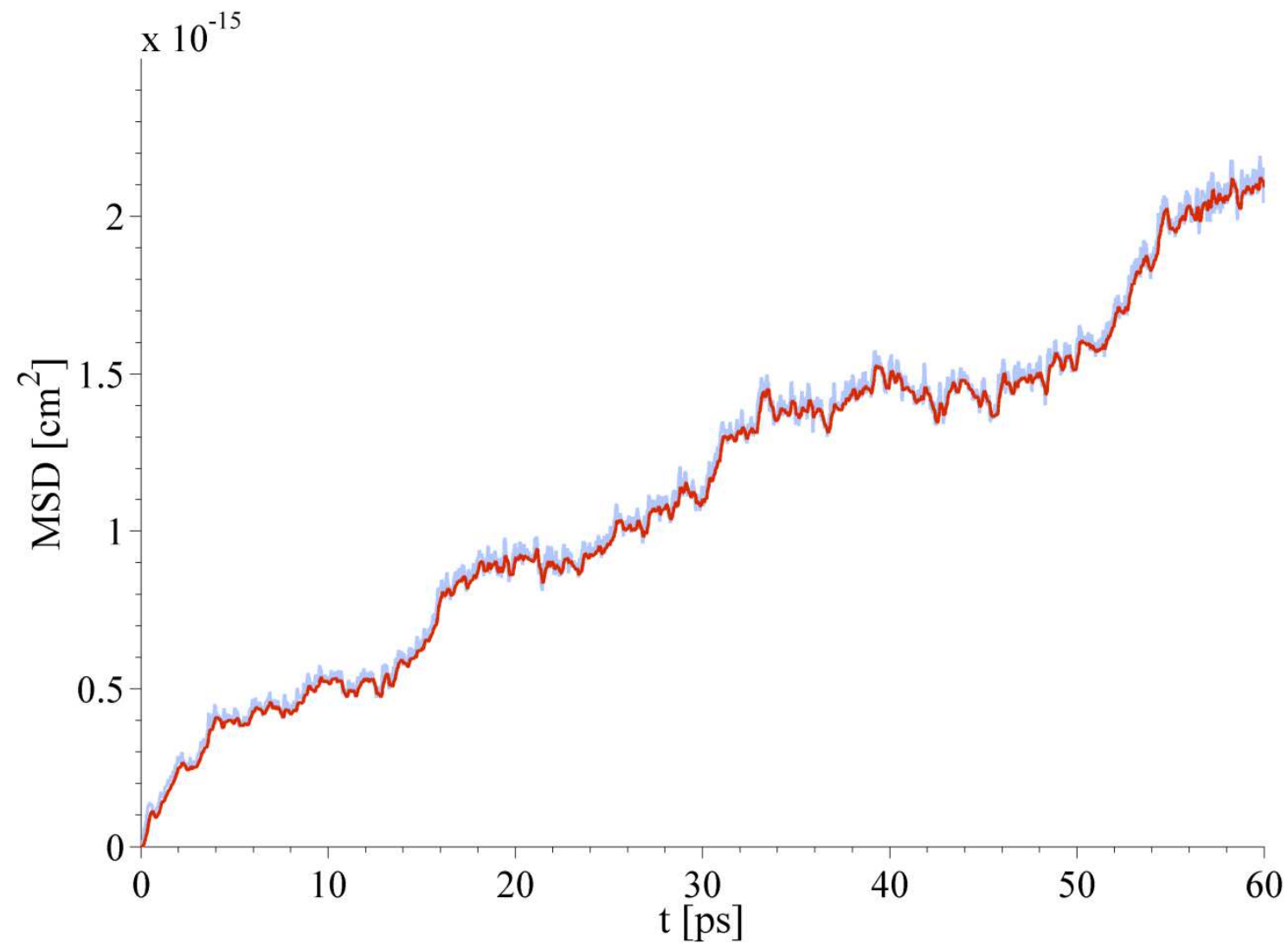

Fig. 3. MSD evolution for molecular dynamic simulation at $1173 \mathrm{~K}$ for unfiltered data (blue) and data processed by the low-pass filter (red). 


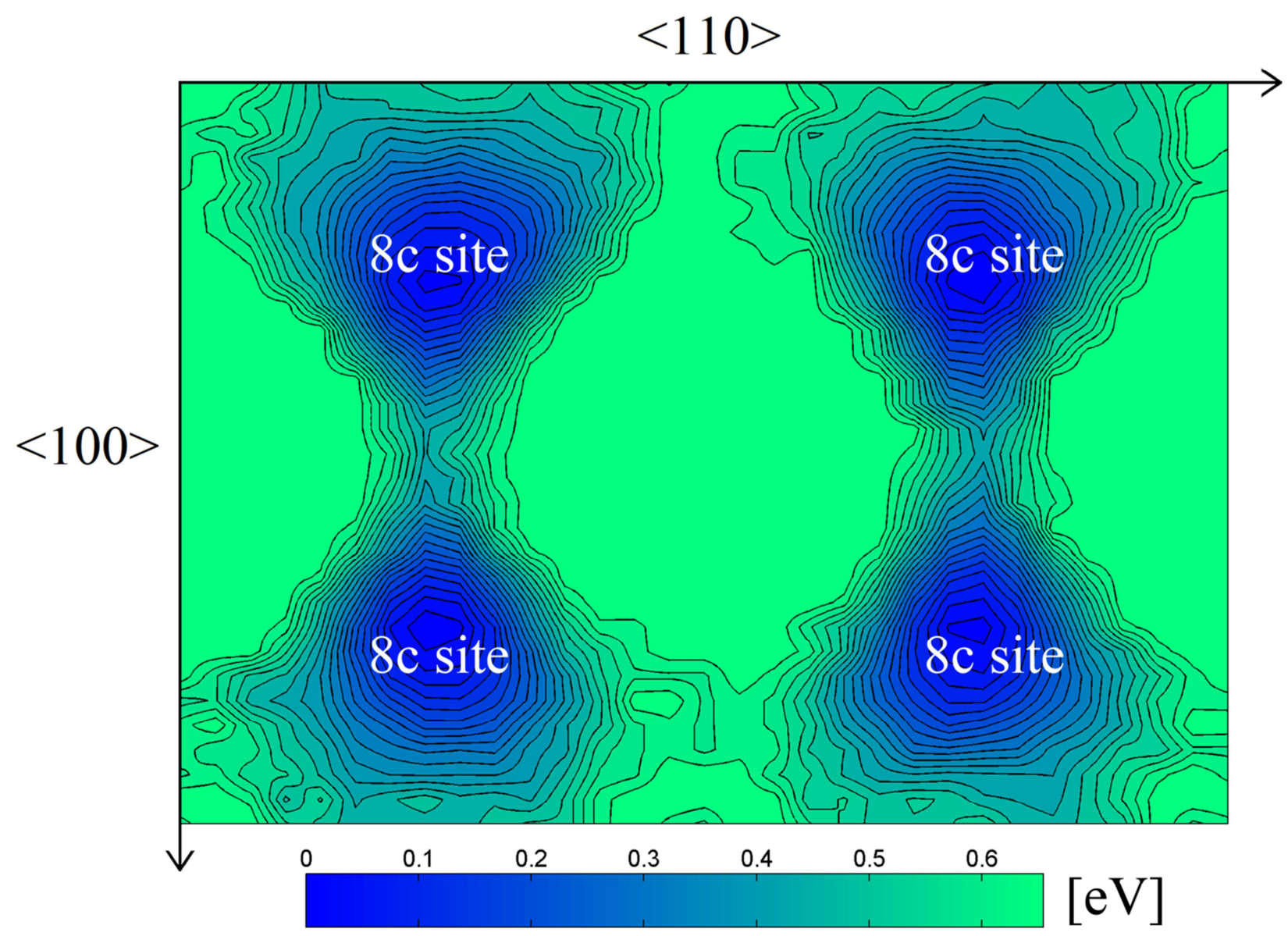

Fig. 4. Anion energy distribution landscape representation in the <110> plane at $1073 \mathrm{~K}$. 

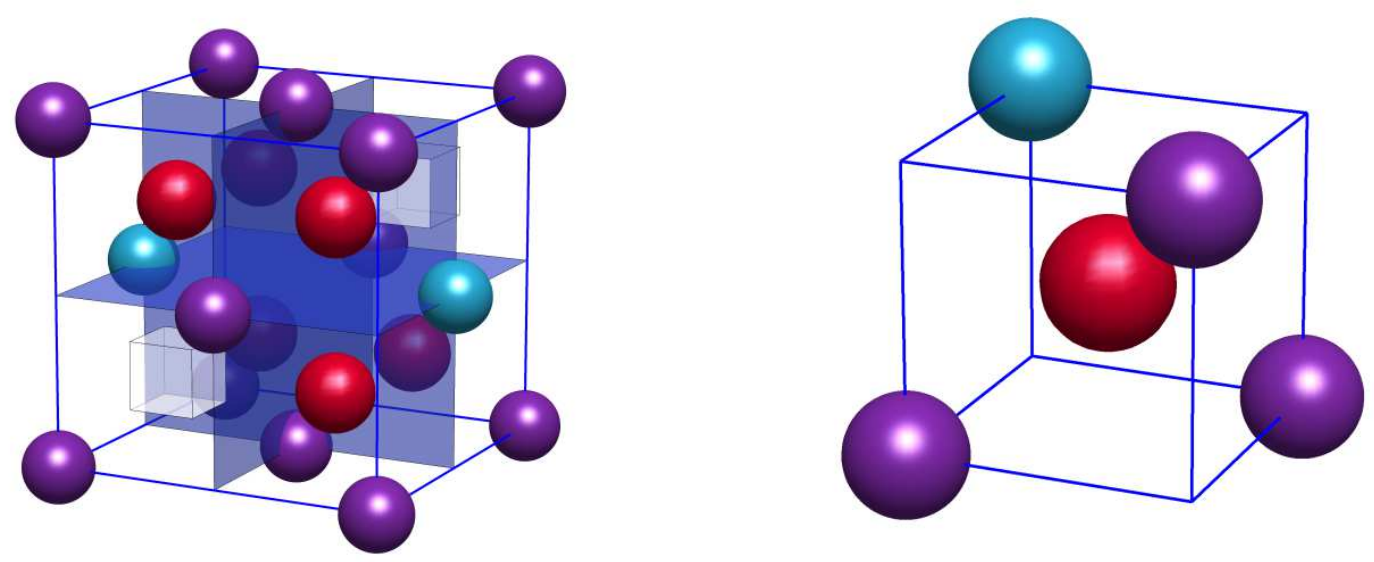

Fig. 5. (a) Single unit cell model for $\delta$ - $\mathrm{Bi}_{3} \mathrm{YO}_{6}$ divided into 8 octants and (b) an example octant with $n=1$ (one yttrium atom). Spheres represent bismuth (violet), yttrium (blue) and oxygen (red) atoms and the semi-transparent cube represents an oxide ion vacancy. 


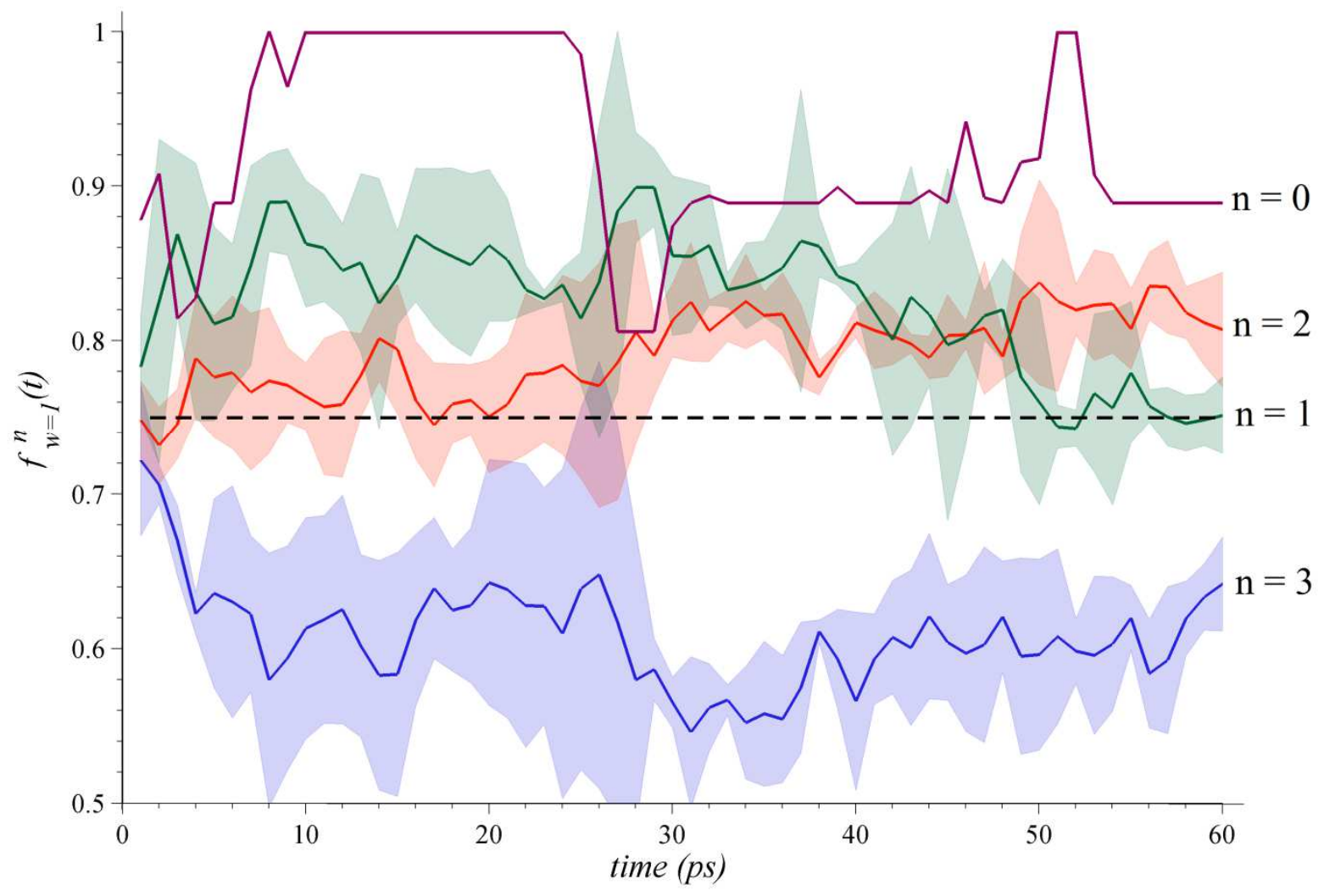

Fig. 6. The average oxide ion site occupancy $f_{w=1}^{n}(t)$ of octants of type $n$ over a $w=1$ ps time window at $1073 \mathrm{~K}$, averaged over three parallel runs. The standard deviation of the occupancy is marked by the shaded regions. The dashed line represents the nominal stoichiometric occupancy of 0.75 . 


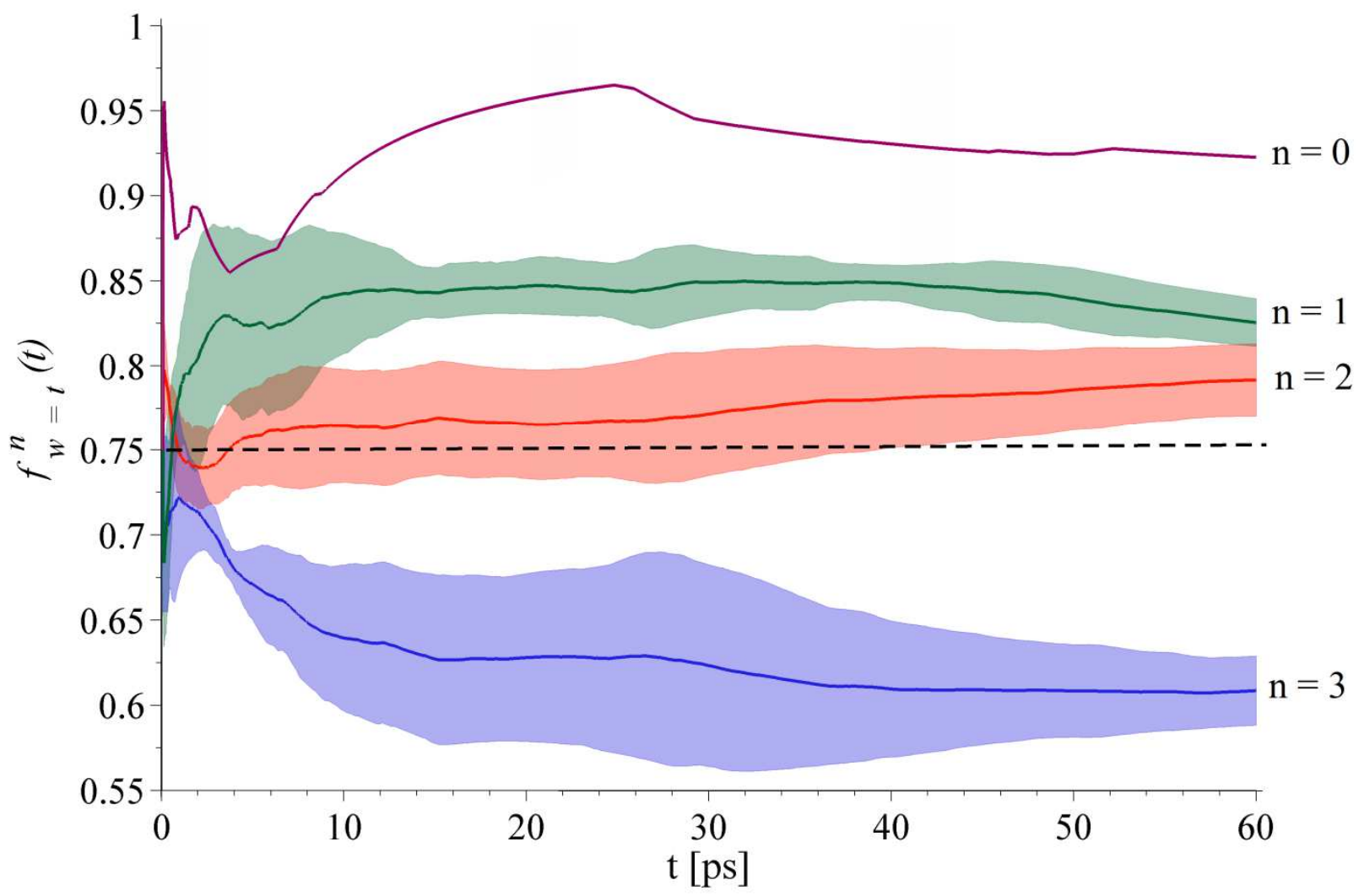

Fig. 7. The average oxide ion site occupancy $f_{w=t}^{n}(t)$ of octants of type $n$ over a time window $w=t$ at $1073 \mathrm{~K}$, averaged over three parallel runs. The standard deviation of the occupancy is marked by the shaded regions. The dashed line represents the nominal stoichiometric occupancy of 0.75 . 


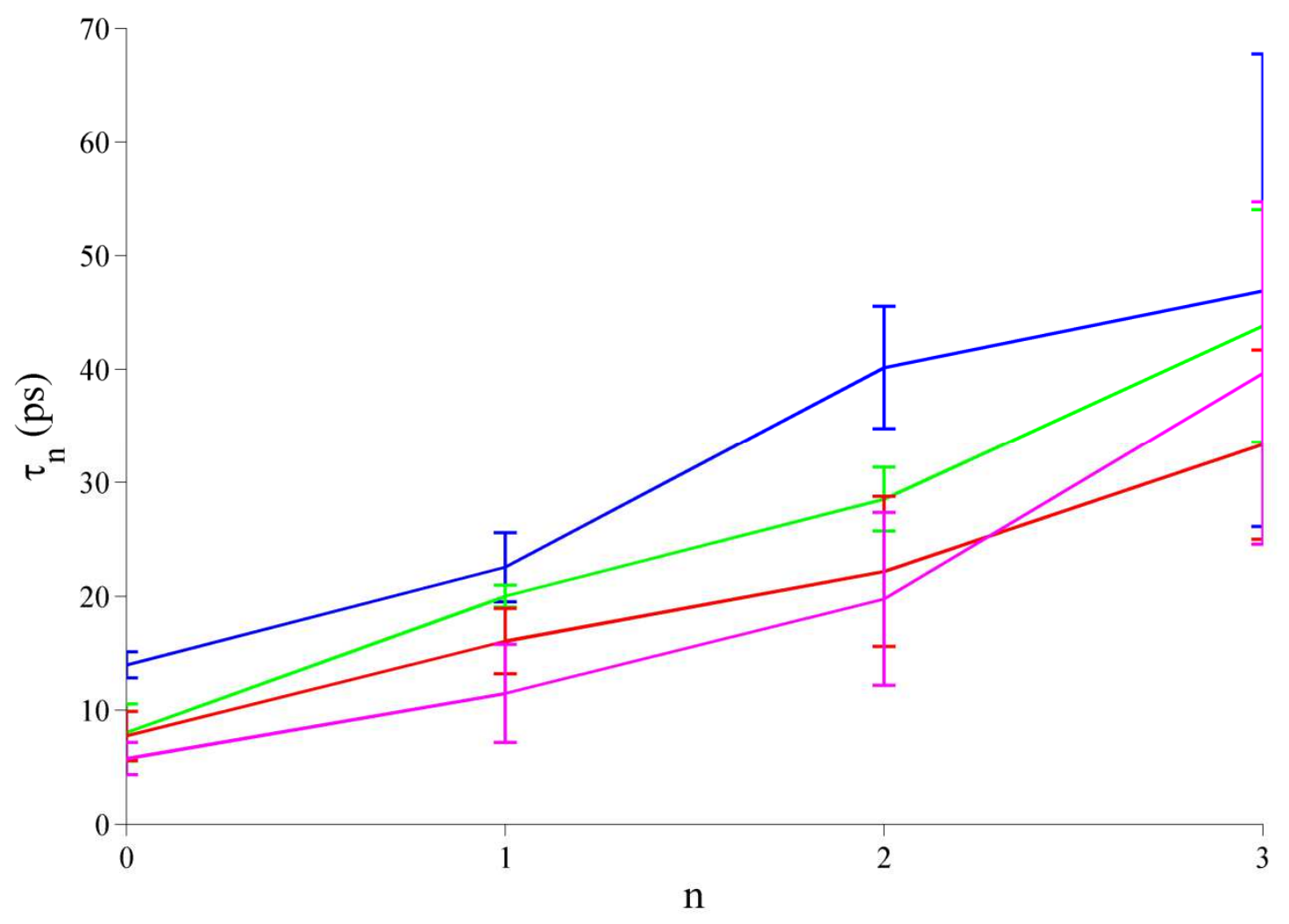



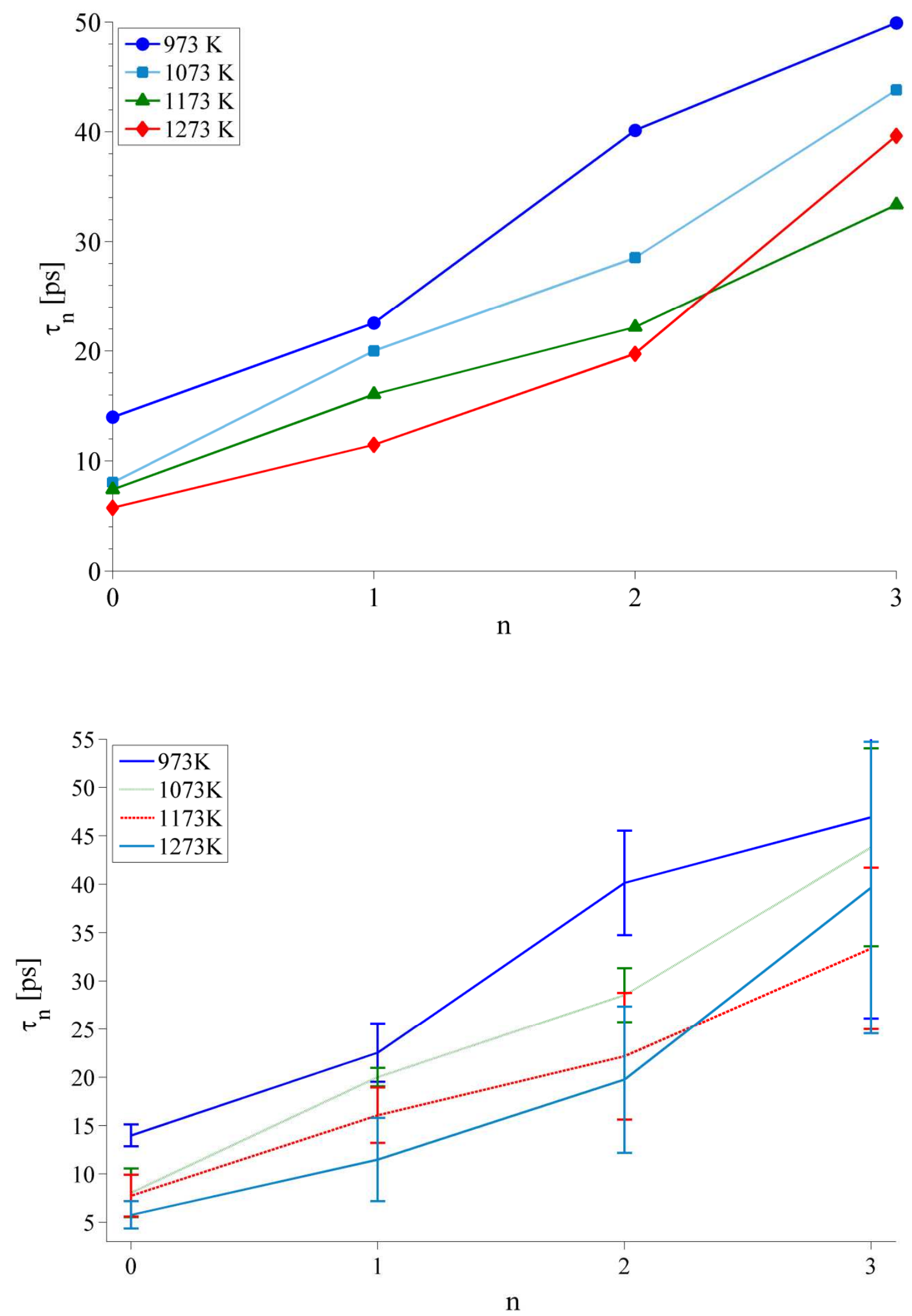
Fig. 8. Average residence time $\tau_{n}$ of oxide ions in octants of type $n$ at different temperatures.

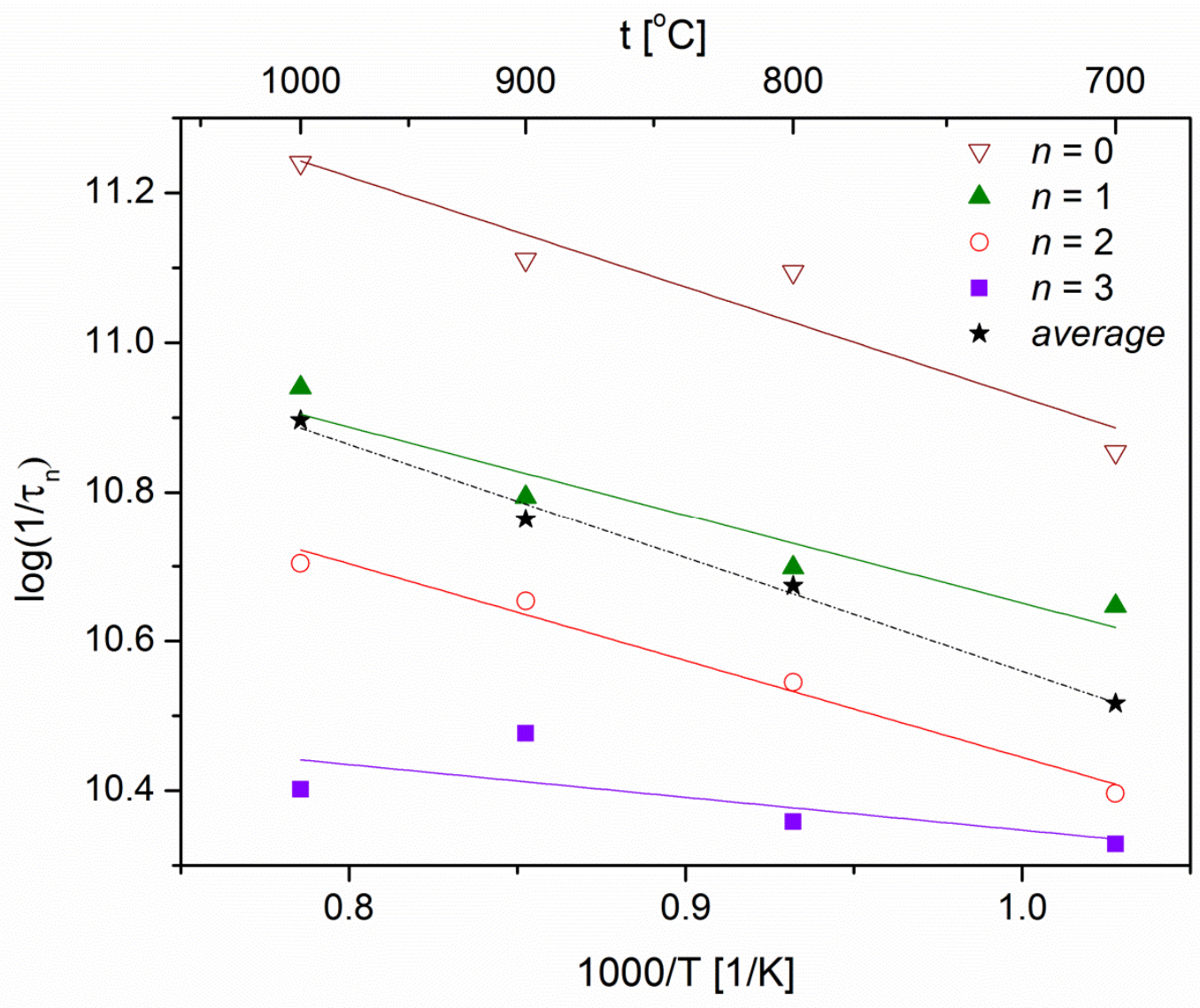

Fig. 9. Arrhenius type plots of $1 / \tau_{n}$ for individual octant types $(n)$. 